\title{
Iron-Catalyzed Arylmagnesiation of Aryl(alkyl)acetylenes in the Presence of an $N$-Heterocyclic Carbene Ligand
}

\author{
Takafumi Yamagami, Ryo Shintani, Eiji Shirakawa,* and Tamio Hayashi*
}

Department of Chemistry, Graduate School of Science, Kyoto University, Sakyo, Kyoto 606-8502, Japan

\section{Supporting Information}

\section{General}

All air- and moisture-sensitive manipulations were carried out with standard Schlenk techniques under nitrogen or in a glove box under argon. THF was purified by passing through a neutral alumina column under nitrogen. 1-Hexyne (Wako Chemicals), phenylacetylene (Wako Chemicals), 4-methyl-1-pentyne (TCI), 3-methyl-1-butyne (Wako Chemicals), 3-butyn-1-ol (Aldrich), iodomethane (Wako Chemical), 2-iodoaniline (Wako Chemicals), 3-iodoaniline (Wako Chemicals), 4-iodoaniline (Wako Chemicals), 1-chloro-4-iodobenzene (TCI), 2-bromoanisole (TCI), 3-bromoanisole (TCI), 4-bromoanisole (TCI), 4-bromotoluene (Wako Chemicals), 1-bromo-3,5-dimethylbenzene (Wako Chemicals), 1-bromo-4-fluorobenzene (Wako Chemicals), 1-bromonaphthalene (TCI), 1-phenyl-1-butyne (Wako Chemicals), Mg (Wako Chemicals), 1,2-dibromomethane (TCI), $\mathrm{Et}_{3} \mathrm{~N}$ (Wako Chemicals), $\mathrm{D}_{2} \mathrm{O}$ (Cambridge Isotope Laboratories, Inc.), allyl bromide (TCI), benzyl bromide (TCI), $\mathrm{ZnCl}_{2}$ (Wako Chemicals), I ${ }_{2}$ (Wako Chemicals), CuI (Wako Chemicals), and iodobenzene (TCI) were used as received.

$\mathrm{Fe}(\mathrm{acac})_{3},{ }_{1} \mathrm{NiCl}_{2}\left(\mathrm{PPh}_{3}\right)_{2},{ }^{2} \mathrm{Pd}\left(\mathrm{PPh}_{3}\right)_{4},{ }^{3}$ and $\operatorname{IPr}^{4}$ were synthesized following the literature procedures.

All other chemicals and solvents were purchased from Aldrich, Wako Chemicals, TCI, or Kanto Chemicals and used as received.

\section{Preparation of Substrates}

\section{General Procedure for the Preparation of Aryl(alkyl)acetylenes}

1-Alkyne (22 mmol) was added to a solution of aryl iodide (20 mmol), $\mathrm{Pd}\left(\mathrm{PPh}_{3}\right)_{4}(232$ $\mathrm{mg}, 0.20 \mathrm{mmol})$, and $\mathrm{CuI}(174 \mathrm{mg}, 1.8 \mathrm{mmol})$ in $\mathrm{Et}_{3} \mathrm{~N}(30 \mathrm{~mL})$ at $60^{\circ} \mathrm{C}$. The resulting mixture was stirred at $60^{\circ} \mathrm{C}$ for $12-24 \mathrm{~h}$, passed through a pad of silica gel with EtOAc, and concentrated under vacuum. The residue was chromatographed on silica gel with hexane to afford the corresponding alkynes as an oil.

\section{General Procedure for the Preparation of Arylmagnesium Bromides}

Aryl bromide solution ( $5 \mathrm{mmol}$ in THF $(3 \mathrm{~mL})$ ) was added dropwise to a suspension of $\mathrm{Mg}$ activated by 1,2-dibromoethane in THF $(1.5 \mathrm{~mL})$ at room temperature. The reaction mixture was stirred at room temperature for $2 \mathrm{~h}$. The concentration was determined by acid-base titration with methyl orange (typically $0.88-1.11 \mathrm{M}$ ).

\footnotetext{
${ }^{1}$ Charles, R. G.; Pawlikowski, M. A. J. Phys. Chem. 1958, 62, 440.

2 Venanzi, L. M. J. Chem. Soc. 1958, 719.

${ }^{3}$ Couison, D. R. Inorg. Synth. 1972, 13, 121.

${ }^{4}$ Jafarpour, L.; Stevens, E. D.; Nolan, S. P. J. Organomet. Chem. 2000, 606, 49.
} 


\section{Catalytic Reactions}

A Typical Procedure for the Arylmagnesiation of Alkynes (entry 1 in Table 1).

A solution of $\mathrm{Fe}(\mathrm{acac})_{3}(3.5 \mathrm{mg}, 9.9 \mu \mathrm{mol})$ and $\operatorname{IPr}(15.6 \mathrm{mg}, 40.0 \mu \mathrm{mol})$ in THF $(1.0$ $\mathrm{mL}$ ) was stirred at room temperature for $5 \mathrm{~min}$. 1-Phenyl-1-hexyne (1a, $31.6 \mathrm{mg}$, $0.200 \mathrm{mmol})$ and 4-methoxyphenylmagnesium bromide $(\mathbf{2 m}, 0.38 \mathrm{~mL}, 0.40 \mathrm{mmol} ; 1.06$ $\mathrm{M}$ solution in THF) were added, and the mixture was stirred at $60{ }^{\circ} \mathrm{C}$ for $16 \mathrm{~h}$. The reaction was quenched with water $(80 \mu \mathrm{L})$, passed through a pad of silica gel with $\mathrm{Et}_{2} \mathrm{O}$, and concentrated under vacuum. The residue was purified by silica gel preparative TLC with Hexane $/ \mathrm{Et}_{2} \mathrm{O}(95 / 5)$ to give product (3am, $48.7 \mathrm{mg}, 0.183 \mathrm{mmol}$; 91\% yield).

\section{General Procedure for Table 2.}

A solution of $\mathrm{Fe}(\mathrm{acac})_{3}(3.5 \mathrm{mg}, 9.9 \mu \mathrm{mol})$ and $\operatorname{IPr}(15.6 \mathrm{mg}, 40.0 \mu \mathrm{mol})$ in THF (1.0 $\mathrm{mL}$ ) was stirred at room temperature for $5 \mathrm{~min}$. An alkyne $(\mathbf{1}, 0.20 \mathrm{mmol})$ and $\operatorname{ArMgBr}(2,0.36-0.46 \mathrm{~mL}, 0.40 \mathrm{mmol}$; $0.88-1.11 \mathrm{M}$ solution in THF) were added, and the mixture was stirred at $60{ }^{\circ} \mathrm{C}$ for $16 \mathrm{~h}$. The reaction was quenched with water $(80$ $\mu \mathrm{L}$ ), passed through a pad of silica gel with $\mathrm{Et}_{2} \mathrm{O}$, and concentrated under vacuum. The residue was purified by silica gel preparative TLC with $\mathrm{Hexane}_{2} / \mathrm{Et}_{2} \mathrm{O}(95 / 5)$ to give product 3.

3am. 2-(4-Methoxyphenyl)-1-phenyl-1-hexene (Colorless oil. 91\% yield; entry 1)

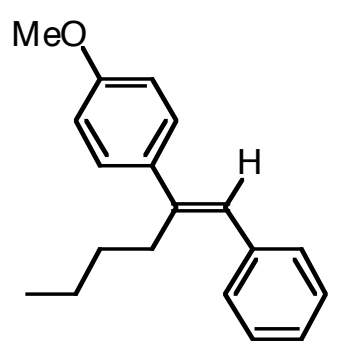

(E)-3am; ${ }^{1} \mathrm{H}$ NMR $\left(\mathrm{CDCl}_{3}\right): \delta 7.39\left(\mathrm{~d},{ }^{3} \mathrm{~J}_{\mathrm{HH}}=8.2 \mathrm{~Hz}, 2 \mathrm{H}\right), 7.35(\mathrm{t}$, $\left.{ }^{3} J_{\mathrm{HH}}=7.6 \mathrm{~Hz}, 2 \mathrm{H}\right), 7.30\left(\mathrm{~d},{ }^{3} J_{\mathrm{HH}}=8.2 \mathrm{~Hz}, 2 \mathrm{H}\right), 7.22\left(\mathrm{t},{ }^{3} J_{\mathrm{HH}}=7.1\right.$ $\mathrm{Hz}, 1 \mathrm{H}), 6.90\left(\mathrm{~d},{ }^{3} J_{\mathrm{HH}}=8.5 \mathrm{~Hz}, 2 \mathrm{H}\right), 6.64(\mathrm{~s}, 1 \mathrm{H}), 3.82(\mathrm{~s}, 3 \mathrm{H})$, $2.67\left(\mathrm{t},{ }^{3} J_{\mathrm{HH}}=7.9 \mathrm{~Hz}, 2 \mathrm{H}\right), 1.41$ (quint, $\left.{ }^{3} J_{\mathrm{HH}}=7.6 \mathrm{~Hz}, 2 \mathrm{H}\right), 1.32$ (sextet, $\left.{ }^{3} J_{\mathrm{HH}}=7.3 \mathrm{~Hz}, 2 \mathrm{H}\right), 0.85\left(\mathrm{t},{ }^{3} J_{\mathrm{HH}}=7.1 \mathrm{~Hz}, 3 \mathrm{H}\right)$. The NOE was observed at ortho protons of 4-methoxyphenyl group (13\%) and ortho protons of phenyl group (10\%) on irradiation at 6.64 ppm $(=\mathrm{CH}-)$. For $(Z)$ isomer, olefin proton appears at $6.39 \mathrm{ppm}$. ${ }^{13} \mathrm{C}$ NMR $\left(\mathrm{CDCl}_{3}\right): \delta 159.1,142.9,138.7,135.7,128.9,128.3$, 127.8, 126.9, 126.4, 113.9, 55.4, 31.2, 30.0, 22.9, 14.0. Anal. Calcd for $\mathrm{C}_{19} \mathrm{H}_{22} \mathrm{O}: \mathrm{C}, 85.67 ; \mathrm{H}, 8.32$. Found: $\mathrm{C}, 85.58 ; \mathrm{H}, 8.41$.

3bm. 2-(4-Methoxyphenyl)-1-phenyl-1-butene (Colorless oil. 91\% yield; entry 2)

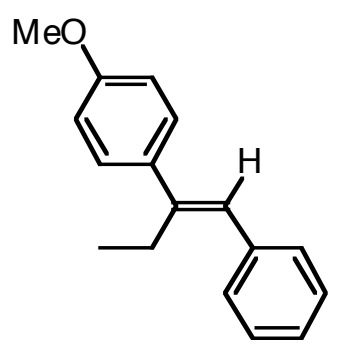

(E)-3bm; ${ }^{1} \mathrm{H}$ NMR $\left(\mathrm{CDCl}_{3}\right): \delta 7.40\left(\mathrm{~d},{ }^{3} J_{\mathrm{HH}}=8.4 \mathrm{~Hz}, 2 \mathrm{H}\right), 7.35(\mathrm{t}$, $\left.{ }^{3} J_{\mathrm{HH}}=7.6 \mathrm{~Hz}, 2 \mathrm{H}\right), 7.31\left(\mathrm{~d},{ }^{3} J_{\mathrm{HH}}=7.0 \mathrm{~Hz}, 2 \mathrm{H}\right), 7.23\left(\mathrm{t},{ }^{3} J_{\mathrm{HH}}=7.2\right.$ $\mathrm{Hz}, 1 \mathrm{H}), 6.90\left(\mathrm{~d},{ }^{3} J_{\mathrm{HH}}=8.5 \mathrm{~Hz}, 2 \mathrm{H}\right), 6.64(\mathrm{~s}, 1 \mathrm{H}), 3.82(\mathrm{~s}, 3 \mathrm{H})$, $2.71\left(\mathrm{q},{ }^{3} J_{\mathrm{HH}}=7.5 \mathrm{~Hz}, 2 \mathrm{H}\right), 1.06\left(\mathrm{t},{ }^{3} J_{\mathrm{HH}}=7.5 \mathrm{~Hz}, 3 \mathrm{H}\right)$. For $(Z)$ isomer, olefin proton appears at $6.39 \mathrm{ppm} .{ }^{13} \mathrm{C} \mathrm{NMR}\left(\mathrm{CDCl}_{3}\right): \delta$ $159.1,144.0,138.7,135.2,128.8,128.4,127.8,126.5,126.5$, 113.9, 55.4, 23.3, 13.7. Anal. Calcd for $\mathrm{C}_{17} \mathrm{H}_{18} \mathrm{O}: \mathrm{C}, 85.67 ; \mathrm{H}$, 7.61. Found: C, 85.73; H, 7.67. 
3cm. 2-(4-Methoxyphenyl)-1-phenyl-4-methyl-1-pentene (Colorless oil. 87\% yield; entry 3$)$

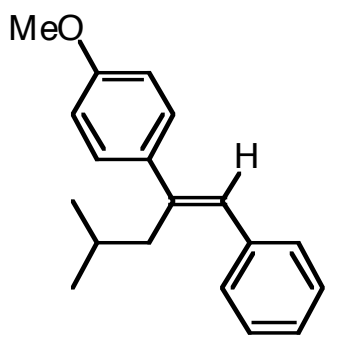

(E)-3cm; ${ }^{1} \mathrm{H}$ NMR $\left(\mathrm{CDCl}_{3}\right): \delta 7.37\left(\mathrm{~d},{ }^{3} \mathrm{~J}_{\mathrm{HH}}=8.2 \mathrm{~Hz}, 2 \mathrm{H}\right), 7.33(\mathrm{t}$, $\left.{ }^{3} J_{\mathrm{HH}}=7.3 \mathrm{~Hz}, 2 \mathrm{H}\right), 7.30\left(\mathrm{~d},{ }^{3} J_{\mathrm{HH}}=6.7 \mathrm{~Hz}, 2 \mathrm{H}\right), 7.22\left(\mathrm{t},{ }^{3} J_{\mathrm{HH}}=6.6\right.$ $\mathrm{Hz}, 1 \mathrm{H}), 6.89\left(\mathrm{~d},{ }^{3} J_{\mathrm{HH}}=8.5 \mathrm{~Hz}, 2 \mathrm{H}\right), 6.67(\mathrm{~s}, 1 \mathrm{H}), 3.82(\mathrm{~s}, 3 \mathrm{H})$, $2.58\left(\mathrm{~d},{ }^{3} J_{\mathrm{HH}}=7.3 \mathrm{~Hz}, 2 \mathrm{H}\right), 1.69$ (heptet, $\left.{ }^{3} J_{\mathrm{HH}}=6.8 \mathrm{~Hz}, 1 \mathrm{H}\right), 0.81$ $\left(\mathrm{d},{ }^{3} J_{\mathrm{HH}}=6.4 \mathrm{~Hz}, 6 \mathrm{H}\right)$. For $(Z)$ isomer, olefin proton appears at $6.37 \mathrm{ppm} .{ }^{13} \mathrm{C}$ NMR $\left(\mathrm{CDCl}_{3}\right): \delta 159.0,142.3,138.7,136.1$, $129.1,128.5,128.3,127.9,126.4,113.8,55.4,38.7,27.3,22.6$. Anal. Calcd for $\mathrm{C}_{19} \mathrm{H}_{22} \mathrm{O}$ : C, 85.67; H, 8.32. Found: C, 85.70; H, 8.38.

3dm. 2-(4-Methoxyphenyl)-1-phenyl-3-methyl-1-butene (Colorless oil. 84\% yield; entry 4)

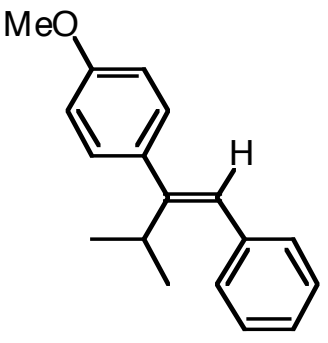

(E)-3dm; ${ }^{1} \mathrm{H}$ NMR $\left(\mathrm{CDCl}_{3}\right): \delta 7.35\left(\mathrm{t},{ }^{3} J_{\mathrm{HH}}=7.5 \mathrm{~Hz}, 2 \mathrm{H}\right), 7.32$ $\left(\mathrm{d},{ }^{3} J_{\mathrm{HH}}=7.0 \mathrm{~Hz}, 2 \mathrm{H}\right), 7.25-7.19(\mathrm{~m}, 1 \mathrm{H}), 7.21\left(\mathrm{~d},{ }^{3} J_{\mathrm{HH}}=8.8 \mathrm{~Hz}\right.$, $2 \mathrm{H}), 6.87\left(\mathrm{~d},{ }^{3} J_{\mathrm{HH}}=8.8 \mathrm{~Hz}, 2 \mathrm{H}\right), 6.33(\mathrm{~s}, 1 \mathrm{H}), 3.82(\mathrm{~s}, 3 \mathrm{H}), 3.27$ (heptet, $\left.{ }^{3} J_{\mathrm{HH}}=6.9 \mathrm{~Hz}, 1 \mathrm{H}\right), 1.05\left(\mathrm{~d},{ }^{3} J_{\mathrm{HH}}=7.0 \mathrm{~Hz}, 6 \mathrm{H}\right)$. For $(Z)$ isomer, olefin proton appears at $6.37 \mathrm{ppm} .{ }^{13} \mathrm{C} \mathrm{NMR}\left(\mathrm{CDCl}_{3}\right)$ : $\delta 158.6,149.5,138.3,135.1,130.1,129.1,129.0,128.3,126.5$, 113.1, 55.4, 29.5, 22.1. Anal. Calcd for $\mathrm{C}_{18} \mathrm{H}_{20} \mathrm{O}: \mathrm{C}, 85.67 ; \mathrm{H}$, 7.99. Found: C, 85.97; H, 8.13.

3an. 2-(4-Methylphenyl)-1-phenyl-1-hexene (Colorless oil. 93\% yield; entry 5)

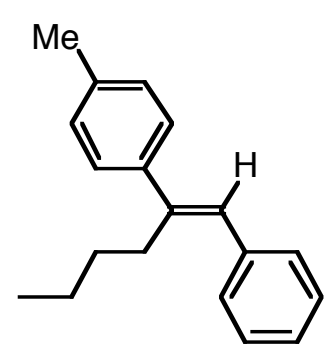

(E)-3an; ${ }^{1} \mathrm{H}$ NMR $\left(\mathrm{CDCl}_{3}\right): \delta 7.35\left(\mathrm{t},{ }^{3} J_{\mathrm{HH}}=7.9 \mathrm{~Hz}, 2 \mathrm{H}\right), 7.33(\mathrm{~d}$, $\left.{ }^{3} J_{\mathrm{HH}}=7.9 \mathrm{~Hz}, 2 \mathrm{H}\right), 7.30\left(\mathrm{~d},{ }^{3} J_{\mathrm{HH}}=7.9 \mathrm{~Hz}, 2 \mathrm{H}\right), 7.22\left(\mathrm{t},{ }^{3} J_{\mathrm{HH}}=6.8\right.$ $\mathrm{Hz}, 1 \mathrm{H}), 6.90\left(\mathrm{~d},{ }^{3} J_{\mathrm{HH}}=8.5 \mathrm{~Hz}, 2 \mathrm{H}\right), 6.67(\mathrm{~s}, 1 \mathrm{H}), 2.68\left(\mathrm{t},{ }^{3} J_{\mathrm{HH}}=\right.$ $7.7 \mathrm{~Hz}, 2 \mathrm{H}), 2.36(\mathrm{~s}, 3 \mathrm{H}), 1.41$ (quint, $\left.{ }^{3} J_{\mathrm{HH}}=7.7 \mathrm{~Hz}, 2 \mathrm{H}\right), 1.31$ (sextet, $\left.{ }^{3} J_{\mathrm{HH}}=7.3 \mathrm{~Hz}, 2 \mathrm{H}\right), 0.84\left(\mathrm{t},{ }^{3} J_{\mathrm{HH}}=7.3 \mathrm{~Hz}, 3 \mathrm{H}\right)$. For $(Z)$ isomer, olefin proton appears at $6.40 \mathrm{ppm} .{ }^{13} \mathrm{C} \mathrm{NMR}\left(\mathrm{CDCl}_{3}\right)$ : $\delta 143.4,140.4,138.6,137.0,129.2,128.9,128.3,127.5,126.6$, 126.5, 31.1, 30.0, 23.0, 21.2, 14.0. Anal. Calcd for $\mathrm{C}_{19} \mathrm{H}_{22}$ : C, 91.14; H, 8.86. Found: C, 91.35; H, 8.82.

3en. 1-(4-Methoxyphenyl)-2-(4-methylphenyl)-1-hexene (Pale yellow oil. 80\% yield; entry 6)

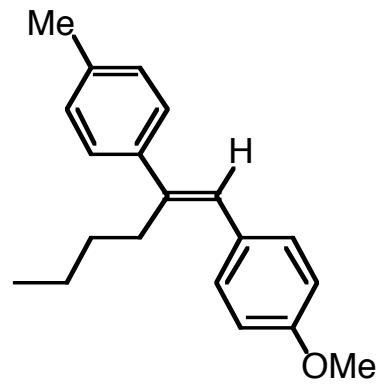

(E)-3en; ${ }^{1} \mathrm{H}$ NMR $\left(\mathrm{CDCl}_{3}\right): \delta 7.34\left(\mathrm{~d},{ }^{3} \mathrm{~J}_{\mathrm{HH}}=7.9 \mathrm{~Hz}, 2 \mathrm{H}\right), 7.25$ $\left(\mathrm{d},{ }^{3} J_{\mathrm{HH}}=8.8 \mathrm{~Hz}, 2 \mathrm{H}\right), 7.15\left(\mathrm{~d},{ }^{3} J_{\mathrm{HH}}=8.5 \mathrm{~Hz}, 2 \mathrm{H}\right), 6.89\left(\mathrm{~d},{ }^{3} J_{\mathrm{HH}}\right.$ $=8.5 \mathrm{~Hz}, 2 \mathrm{H}), 6.61(\mathrm{~s}, 1 \mathrm{H}), 3.81(\mathrm{~s}, 3 \mathrm{H}), 2.68\left(\mathrm{t},{ }^{3} J_{\mathrm{HH}}=7.9 \mathrm{~Hz}\right.$, $2 \mathrm{H}$ ), $2.36\left(\mathrm{~s}, 3 \mathrm{H}\right.$ ), 1.41 (quint, ${ }^{3} J_{\mathrm{HH}}=7.7 \mathrm{~Hz}, 2 \mathrm{H}$ ), 1.33 (sextet, $\left.{ }^{3} J_{\mathrm{HH}}=7.3 \mathrm{~Hz}, 2 \mathrm{H}\right), 0.85\left(\mathrm{t},{ }^{3} J_{\mathrm{HH}}=7.3 \mathrm{~Hz}, 3 \mathrm{H}\right)$. For $(Z)$ isomer, olefin proton appears at $6.33 \mathrm{ppm} .{ }^{13} \mathrm{C} \mathrm{NMR}\left(\mathrm{CDCl}_{3}\right): \delta$ $158.3,142.0,140.6,136.8,130.2,130.1,129.2$, 127.1, 126.5, $113.8,55.4,31.1,30.0,23.0,21.2,14.1$. Anal. Calcd for $\mathrm{C}_{20} \mathrm{H}_{24} \mathrm{O}: \mathrm{C}, 85.67 ; \mathrm{H}, 8.63$. Found: $\mathrm{C}, 85.83 ; \mathrm{H}, 8.90$. 
3fn. 1-(3-Methoxyphenyl)-2-(4-methylphenyl)-1-hexene (Colorless oil. 87\% yield; entry 7)

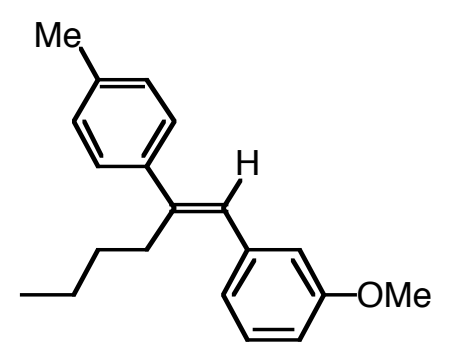

(E)-3fn; ${ }^{1} \mathrm{H}$ NMR $\left(\mathrm{CDCl}_{3}\right): \delta 7.35\left(\mathrm{~d},{ }^{3} J_{\mathrm{HH}}=7.9 \mathrm{~Hz}, 2 \mathrm{H}\right)$, $7.26\left(\mathrm{t},{ }^{3} J_{\mathrm{HH}}=7.9 \mathrm{~Hz}, 1 \mathrm{H}\right), 7.16\left(\mathrm{~d},{ }^{3} J_{\mathrm{HH}}=8.2 \mathrm{~Hz}, 2 \mathrm{H}\right), 6.90$ $\left(\mathrm{d},{ }^{3} J_{\mathrm{HH}}=7.3 \mathrm{~Hz}, 1 \mathrm{H}\right), 6.86(\mathrm{~s}, 1 \mathrm{H}), 6.79\left(\mathrm{~d},{ }^{3} J_{\mathrm{HH}}=8.2 \mathrm{~Hz}\right.$, $1 \mathrm{H}), 6.64(\mathrm{~s}, 1 \mathrm{H}), 3.80(\mathrm{~s}, 1 \mathrm{H}), 2.69\left(\mathrm{t},{ }^{3} J_{\mathrm{HH}}=7.8 \mathrm{~Hz}, 2 \mathrm{H}\right)$, $2.36(\mathrm{~s}, 3 \mathrm{H}), 1.41$ (quint, ${ }^{3} \mathrm{~J}_{\mathrm{HH}}=7.7 \mathrm{~Hz}, 2 \mathrm{H}$ ), 1.32 (sextet, $\left.{ }^{3} J_{\mathrm{HH}}=7.3 \mathrm{~Hz}, 2 \mathrm{H}\right), 0.84\left(\mathrm{t},{ }^{3} J_{\mathrm{HH}}=7.3 \mathrm{~Hz}, 3 \mathrm{H}\right){ }_{13}$ For $(Z)$ isomer, olefin proton appears at $6.37 \mathrm{ppm} .{ }^{13} \mathrm{C}$ NMR $\left(\mathrm{CDCl}_{3}\right): \delta 159.6,143.6,140.3,140.0,137.0,129.3,129.2$, $127.4,126.6,121.5,114.3,112.2,55.3,31.2,30.2,23.0$, 21.2, 14.1. Anal. Calcd for $\mathrm{C}_{20} \mathrm{H}_{24} \mathrm{O}: \mathrm{C}, 85.67 ; \mathrm{H}, 8.63$. Found: C, 85.54; H, 8.71.

3gn. 1-(2-Methoxyphenyl)-2-(4-methylphenyl)-1-hexene (Colorless oil. 75\% yield; entry 8)

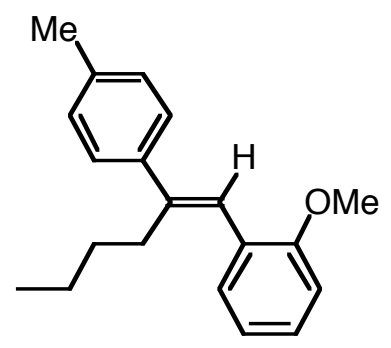

(E)-3gn; ${ }^{1} \mathrm{H}$ NMR $\left(\mathrm{CDCl}_{3}\right): \delta 7.38\left(\mathrm{~d},{ }^{3} J_{\mathrm{HH}}=7.9 \mathrm{~Hz}, 2 \mathrm{H}\right), 7.28$ $\left(\mathrm{d},{ }^{3} J_{\mathrm{HH}}=7.6 \mathrm{~Hz}, 1 \mathrm{H}\right), 7.24\left(\mathrm{t},{ }^{3} J_{\mathrm{HH}}=7.5 \mathrm{~Hz}, 1 \mathrm{H}\right), 7.16\left(\mathrm{~d},{ }^{3} J_{\mathrm{HH}}\right.$ $=7.9 \mathrm{~Hz}, 2 \mathrm{H}), 6.96\left(\mathrm{t},{ }^{3} J_{\mathrm{HH}}=7.5 \mathrm{~Hz}, 1 \mathrm{H}\right), 6.88\left(\mathrm{~d},{ }^{3} J_{\mathrm{HH}}=8.2\right.$ $\mathrm{Hz}, 1 \mathrm{H}), 6.72(\mathrm{~s}, 1 \mathrm{H}), 3.80(\mathrm{~s}, 1 \mathrm{H}), 2.64\left(\mathrm{t},{ }^{3} J_{\mathrm{HH}}=7.9 \mathrm{~Hz}, 2 \mathrm{H}\right)$, $2.36(\mathrm{~s}, 3 \mathrm{H}), 1.39$ (quint, $\left.{ }^{3} J_{\mathrm{HH}}=7.6 \mathrm{~Hz}, 2 \mathrm{H}\right), 1.30$ (sextet, ${ }^{3} J_{\mathrm{HH}}$ $=7.2 \mathrm{~Hz}, 2 \mathrm{H}), 0.83\left(\mathrm{t},{ }^{3} J_{\mathrm{HH}}=7.3 \mathrm{~Hz}, 3 \mathrm{H}\right)$. For $(Z)$ isomer, olefin proton appears at $6.59 \mathrm{ppm} .{ }^{13} \mathrm{C} \mathrm{NMR}\left(\mathrm{CDCl}_{3}\right): \delta$ $157.7,142.8,140.3,130.6,129.8,129.1,129.0,128.1$, 126.7, 123.2, 120.2, 110.6, 55.5, 31.2, 30.1, 23.0, 21.2, 14.0. Anal. Calcd for $\mathrm{C}_{20} \mathrm{H}_{24} \mathrm{O}: \mathrm{C}, 85.67 ; \mathrm{H}, 8.63$. Found: $\mathrm{C}, 85.89 ; \mathrm{H}, 8.65$.

3hm. 1-(4-Chlorophenyl)-2-(4-methoxyphenyl)-1-hexene (Colorless oil. 69\% yield; entry 9)

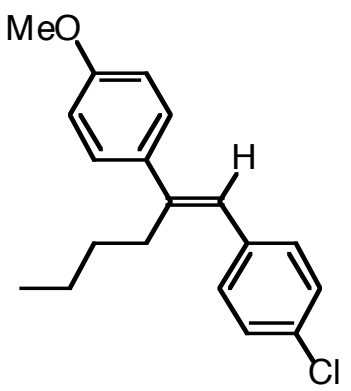

(E)-3hm; ${ }^{1} \mathrm{H}$ NMR $\left(\mathrm{CDCl}_{3}\right): \delta 7.38\left(\mathrm{~d},{ }^{3} \mathrm{~J}_{\mathrm{HH}}=8.8 \mathrm{~Hz}, 2 \mathrm{H}\right), 7.31(\mathrm{~d}$, $\left.{ }^{3} J_{\mathrm{HH}}=8.5 \mathrm{~Hz}, 2 \mathrm{H}\right), 7.22\left(\mathrm{~d},{ }^{3} J_{\mathrm{HH}}=8.2 \mathrm{~Hz}, 2 \mathrm{H}\right), 6.90\left(\mathrm{~d},{ }^{3} J_{\mathrm{HH}}=8.8\right.$ $\mathrm{Hz}, 2 \mathrm{H}), 6.57(\mathrm{~s}, 1 \mathrm{H}), 3.83(\mathrm{~s}, 3 \mathrm{H}), 2.63\left(\mathrm{t},{ }^{3} J_{\mathrm{HH}}=7.9 \mathrm{~Hz}, 2 \mathrm{H}\right)$, 1.39 (quint, ${ }^{3} J_{\mathrm{HH}}=7.7 \mathrm{~Hz}, 2 \mathrm{H}$ ), 1.31 (sextet, ${ }^{3} J_{\mathrm{HH}}=7.4 \mathrm{~Hz}, 2 \mathrm{H}$ ), $0.85\left(\mathrm{t},{ }^{3} J_{\mathrm{HH}}=7.3 \mathrm{~Hz}, 3 \mathrm{H}\right)$. For $(Z)$ isomer, olefin proton appears at $6.33 \mathrm{ppm} .{ }^{13} \mathrm{C} \mathrm{NMR}\left(\mathrm{CDCl}_{3}\right): \delta 159.2,143.6,137.1$, $135.3,130.2,129.7,128.5,127.8,125.6,113.9,55.5,31.1,30.0$, 22.9, 14.0. Anal. Calcd for $\mathrm{C}_{19} \mathrm{H}_{21} \mathrm{ClO}: \mathrm{C}, 75.86 ; \mathrm{H}, 7.04$. Found: C, 75.97; H, 7.15. 
3eo. 2-(3,5-Dimethylphenyl)-1-(4-methoxyphenyl)-1-hexene (Pale yellow oil. 73\% yield; entry 10)

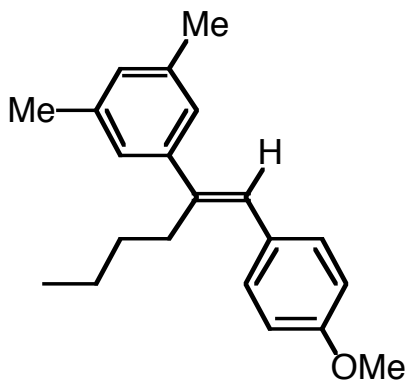

(E)-3eo; ${ }^{1} \mathrm{H}$ NMR $\left(\mathrm{CDCl}_{3}\right): \delta 7.24\left(\mathrm{~d},{ }^{3} \mathrm{~J}_{\mathrm{HH}}=8.5 \mathrm{~Hz}, 2 \mathrm{H}\right), 7.05$ $(\mathrm{s}, 2 \mathrm{H}), 6.91(\mathrm{~s}, 1 \mathrm{H}), 6.89\left(\mathrm{~d},{ }^{3} J_{\mathrm{HH}}=8.8 \mathrm{~Hz}, 2 \mathrm{H}\right), 6.59(\mathrm{~s}, 1 \mathrm{H})$, $3.81(\mathrm{~s}, 3 \mathrm{H}), 2.66\left(\mathrm{t},{ }^{3} J_{\mathrm{HH}}=7.8 \mathrm{~Hz}, 2 \mathrm{H}\right), 2.33(\mathrm{~s}, 6 \mathrm{H}), 1.41$ (quint, ${ }^{3} J_{\mathrm{HH}}=7.6 \mathrm{~Hz}, 2 \mathrm{H}$ ), $1.33\left(\right.$ sextet, ${ }^{3} J_{\mathrm{HH}}=7.2 \mathrm{~Hz}, 2 \mathrm{H}$ ), $0.86\left(\mathrm{t},{ }^{3} J_{\mathrm{HH}}=7.1 \mathrm{~Hz}, 3 \mathrm{H}\right)$. For $(Z)$ isomer, olefin proton appears at $6.30 \mathrm{ppm} .{ }^{13} \mathrm{C}$ NMR $\left(\mathrm{CDCl}_{3}\right): \delta 158.3,143.7$, $142.5,137.8,131.2,130.1,128.8,127.4,124.6,113.8,55.4$, 31.2, 30.2, 23.0, 21.6, 14.1. Anal. Calcd for $\mathrm{C}_{21} \mathrm{H}_{26} \mathrm{O}: \mathrm{C}$, 85.67; H, 8.90. Found: C, 85.40; H, 8.70.

3ap. 2-(3-Methoxyphenyl)-1-phenyl-1-hexene (Colorless oil. 75\% yield; entry 11)

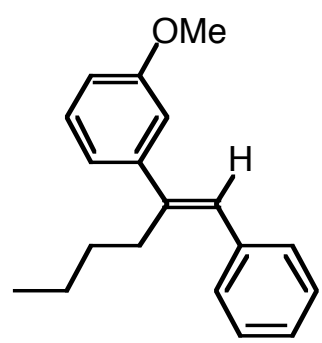

(E)-3ap; ${ }^{1} \mathrm{H}$ NMR $\left(\mathrm{CDCl}_{3}\right): \delta$ 7.38-7.02 (m, 7H), $7.00(\mathrm{~s}, 1 \mathrm{H})$, $6.84\left(\mathrm{dd},{ }^{3} J_{\mathrm{HH}}=8.2 \mathrm{~Hz},{ }^{4} J_{\mathrm{HH}}=2.4 \mathrm{~Hz}, 1 \mathrm{H}\right), 6.70(\mathrm{~s}, 1 \mathrm{H}), 3.84(\mathrm{~s}$, $3 \mathrm{H}), 2.68\left(\mathrm{t},{ }^{3} \mathrm{~J}_{\mathrm{HH}}=7.9 \mathrm{~Hz}, 2 \mathrm{H}\right), 1.42$ (quint, ${ }^{3} \mathrm{~J}_{\mathrm{HH}}=7.7 \mathrm{~Hz}, 2 \mathrm{H}$ ), 1.32 (sextet, $\left.{ }^{3} J_{\mathrm{HH}}=7.3 \mathrm{~Hz}, 2 \mathrm{H}\right), 0.85\left(\mathrm{t},{ }^{3} J_{\mathrm{HH}}=7.2 \mathrm{~Hz}, 3 \mathrm{H}\right)$. For $(Z)$ isomer, olefin proton appears at $6.41 \mathrm{ppm} .{ }^{13} \mathrm{C} \mathrm{NMR}$ $\left(\mathrm{CDCl}_{3}\right): \delta 159.8,145.0,143.4,138.4,129.4,129.1,128.9,128.4$ 126.7, 119.3, 112.8, 112.5, 55.4, 31.1, 30.2, 22.9, 14.0. Anal. Calcd for $\mathrm{C}_{19} \mathrm{H}_{22} \mathrm{O}$ : C, 85.67; H, 8.32. Found: C, 85.76; H, 8.39.

3aq. 2-(2-Methoxyphenyl)-1-phenyl-1-hexene (Colorless oil. 59\% yield; entry 12)

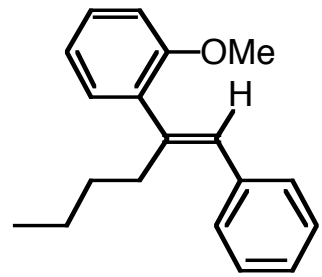

(E)-3aq; ${ }^{1} \mathrm{H}$ NMR $\left(\mathrm{CDCl}_{3}\right): \delta$ 7.36-7.33 (m, 4H), 7.29-7.20 (m, $3 \mathrm{H}), 6.95\left(\mathrm{t},{ }^{3} J_{\mathrm{HH}}=7.5 \mathrm{~Hz}, 1 \mathrm{H}\right), 6.89\left(\mathrm{~d},{ }^{3} J_{\mathrm{HH}}=8.2 \mathrm{~Hz}, 1 \mathrm{H}\right), 6.45$ $(\mathrm{s}, 1 \mathrm{H}), 3.82(\mathrm{~s}, 3 \mathrm{H}), 2.70\left(\mathrm{t},{ }^{3} J_{\mathrm{HH}}=7.6 \mathrm{~Hz}, 2 \mathrm{H}\right), 1.32-1.22(\mathrm{~m}$, $4 \mathrm{H}), 0.79\left(\mathrm{t},{ }^{3} J_{\mathrm{HH}}=6.9 \mathrm{~Hz}, 3 \mathrm{H}\right)$. For $(Z)$ isomer, olefin proton appears at $6.47 \mathrm{ppm} .{ }^{13} \mathrm{C} \mathrm{NMR}\left(\mathrm{CDCl}_{3}\right): \delta 157.0,143.3,138.3$, 133.7, 130.6, 129.2, 129.1, 128.3, 128.2, 126.4, 120.6, 110.9, 55.6, 30.9, 30.8, 22.9, 14.0. Anal. Calcd for $\mathrm{C}_{19} \mathrm{H}_{22} \mathrm{O}: \mathrm{C}, 85.67 ; \mathrm{H}$, 8.32. Found: C, 85.46; H, 8.29.

3er. 2-(4-Fluorophenyl)-1-(4-methoxyphenyl)-1-hexene (Colorless oil. 73\% yield; entry 13)

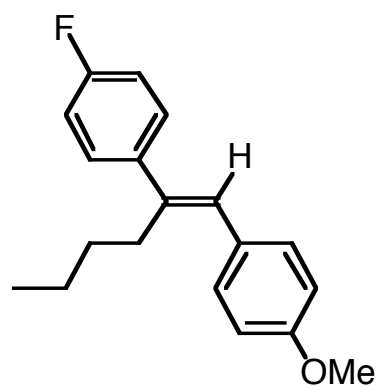

(E)-3er; ${ }^{1} \mathrm{H} \mathrm{NMR}\left(\mathrm{CDCl}_{3}\right): \delta 7.39\left(\mathrm{dd},{ }^{3} J_{\mathrm{HH}}=8.5 \mathrm{~Hz},{ }^{3} J_{\mathrm{HF}}=5.5\right.$ $\mathrm{Hz}, 2 \mathrm{H}), 7.24\left(\mathrm{~d},{ }^{3} \mathrm{~J}_{\mathrm{HH}}=8.5 \mathrm{~Hz}, 2 \mathrm{H}\right), 7.02\left(\mathrm{dd},{ }^{3} \mathrm{~J}_{\mathrm{HH}}=8.5 \mathrm{~Hz}\right.$, $\left.{ }^{3} J_{\mathrm{HF}}=8.5 \mathrm{~Hz}, 2 \mathrm{H}\right), 6.90\left(\mathrm{~d},{ }^{3} J_{\mathrm{HH}}=8.8 \mathrm{~Hz}, 2 \mathrm{H}\right), 6.57(\mathrm{~s}, 1 \mathrm{H})$, $3.82(\mathrm{~s}, 3 \mathrm{H}), 2.66\left(\mathrm{t},{ }^{3} J_{\mathrm{HH}}=7.8 \mathrm{~Hz}, 2 \mathrm{H}\right), 1.43-1.27(\mathrm{~m}, 4 \mathrm{H})$, $0.85\left(\mathrm{t},{ }^{3} J_{\mathrm{HH}}=7.2 \mathrm{~Hz}, 3 \mathrm{H}\right)$. For $(Z)$ isomer, olefin proton appears at $6.37 \mathrm{ppm} .{ }^{13} \mathrm{C} \mathrm{NMR}\left(\mathrm{CDCl}_{3}\right): \delta 162.2\left(\mathrm{~d},{ }^{1} J_{\mathrm{CF}}=\right.$ $244.9 \mathrm{~Hz}), 158.5,141.2,139.5\left(\mathrm{~d},{ }^{4} J_{\mathrm{CF}}=3.1 \mathrm{~Hz}\right), 130.2,130.1$, $128.2\left(\mathrm{~d},{ }^{3} J_{\mathrm{CF}}=8.3 \mathrm{~Hz}\right), 127.8,115.2\left(\mathrm{~d},{ }^{2} J_{\mathrm{CF}}=21.7 \mathrm{~Hz}\right), 113.9$, 55.4, 31.0, 30.2, 22.9, 14.0. Anal. Calcd for $\mathrm{C}_{19} \mathrm{H}_{21} \mathrm{FO}$ : $\mathrm{C}$, 80.25; H, 7.44. Found: C, 80.16; H, 7.56. 
3es. 1-(4-Methoxyphenyl)-2-(1-naphthyl)-1-hexene (yellow oil. 69\% yield; entry 14)

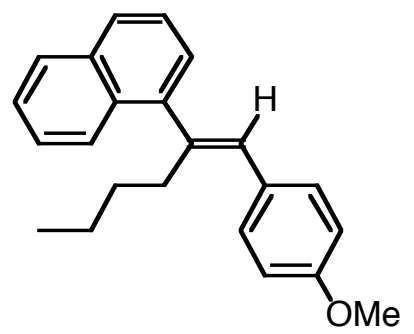

(E)-3es; ${ }^{1} \mathrm{H}$ NMR $\left(\mathrm{CDCl}_{3}\right): \delta 8.10-8.05(\mathrm{~m}, 1 \mathrm{H}), 7.88-7.83(\mathrm{~m}$, $1 \mathrm{H}), 7.77\left(\mathrm{~d},{ }^{3} J_{\mathrm{HH}}=7.9 \mathrm{~Hz}, 1 \mathrm{H}\right), 7.49-7.41(\mathrm{~m}, 3 \mathrm{H}), 7.38-7.32$ $(\mathrm{m}, 3 \mathrm{H}), 6.94\left(\mathrm{~d},{ }^{3} J_{\mathrm{HH}}=8.5 \mathrm{~Hz}, 2 \mathrm{H}\right), 6.46(\mathrm{~s}, 1 \mathrm{H}), 3.83(\mathrm{~s}$, $3 \mathrm{H}), 2.74\left(\mathrm{t},{ }^{3} J_{\mathrm{HH}}=7.9 \mathrm{~Hz}, 2 \mathrm{H}\right), 1.38$ (quint, ${ }^{3} J_{\mathrm{HH}}=7.7 \mathrm{~Hz}$, $2 \mathrm{H}), 1.27\left(\right.$ sextet, $\left.{ }^{3} J_{\mathrm{HH}}=7.3 \mathrm{~Hz}, 2 \mathrm{H}\right), 0.79\left(\mathrm{t},{ }^{3} J_{\mathrm{HH}}=7.2 \mathrm{~Hz}\right.$, $3 \mathrm{H})$. For $(Z)$ isomer, olefin proton appears at $6.63 \mathrm{ppm}$. ${ }^{13} \mathrm{C} \mathrm{NMR}\left(\mathrm{CDCl}_{3}\right): \delta 158.5,142.9,141.4,134.0,132.0,130.7$, $130.1,130.0,128.4,127.1,126.1,125.8,125.7,125.5,125.4$, 113.9, 55.4, 33.4, 30.9, 23.0, 14.0. Anal. Calcd for $\mathrm{C}_{23} \mathrm{H}_{24} \mathrm{O}$ : C, 87.30; H, 7.64. Found: C, 87.54; H, 7.66.

\section{Procedure for the Iron-Catalyzed Arylmagnesiation and Deuteration}

A solution of $\mathrm{Fe}(\mathrm{acac})_{3}(3.5 \mathrm{mg}, 9.9 \mu \mathrm{mol})$ and $\operatorname{IPr}(15.6 \mathrm{mg}, 40.0 \mu \mathrm{mol})$ in THF $(1.0$ $\mathrm{mL})$ was stirred at room temperature for $5 \mathrm{~min}$. 1-Phenyl-1-hexyne (1a, $31.6 \mathrm{mg}$, $0.200 \mathrm{mmol})$ and 4-methoxyphenylmagnesium bromide $(\mathbf{2 m}, 0.38 \mathrm{~mL}, 0.40 \mathrm{mmol} ; 1.06$ $\mathrm{M}$ solution in THF) were added, and the mixture was stirred at $60{ }^{\circ} \mathrm{C}$ for $16 \mathrm{~h}$. After quenching with deuterium oxide $(80 \mu \mathrm{L})$, the reaction mixture was passed through a pad of silica gel with $\mathrm{Et}_{2} \mathrm{O}$, and concentrated under vacuum. The residue was purified by silica gel preparative TLC with Hexane $/ \mathrm{Et}_{2} \mathrm{O}(95 / 5)$ to give product (3am- $d, 49.2 \mathrm{mg}$, 0.184 mmol; $92 \%$ yield ).

3am- $d$. 1-Deuterio-2-(4-methoxyphenyl)-1-phenyl-1-hexene (Colorless oil)

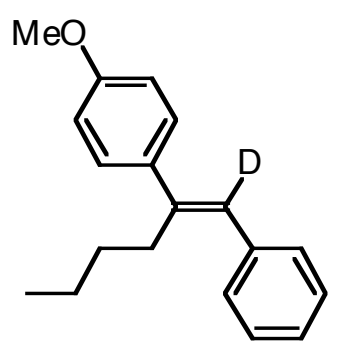

(E)-3am- $d ;{ }^{1} \mathrm{H}$ NMR $\left(\mathrm{CDCl}_{3}\right): \delta 7.39\left(\mathrm{dd},{ }^{3} J_{\mathrm{HH}}=8.8 \mathrm{~Hz}\right.$ and ${ }^{4} J_{\mathrm{HH}}$ $=1.8 \mathrm{~Hz}, 2 \mathrm{H}), 7.37-7.32(\mathrm{~m}, 2 \mathrm{H}), 7.32-7.28(\mathrm{~m}, 2 \mathrm{H}), 7.22(\mathrm{t}$, $\left.{ }^{3} J_{\mathrm{HH}}=7.2 \mathrm{~Hz}, 1 \mathrm{H}\right), 6.90\left(\mathrm{dd},{ }^{3} J_{\mathrm{HH}}=8.5 \mathrm{~Hz}\right.$ and ${ }^{4} J_{\mathrm{HH}}=1.8 \mathrm{~Hz}$, $2 \mathrm{H}), 3.82(\mathrm{~s}, 3 \mathrm{H}), 2.67\left(\mathrm{t},{ }^{3} J_{\mathrm{HH}}=7.9 \mathrm{~Hz}, 2 \mathrm{H}\right), 1.42\left(\right.$ quint, ${ }^{3} J_{\mathrm{HH}}=$ $7.3 \mathrm{~Hz}, 2 \mathrm{H}), 1.32$ (sextet, $\left.{ }^{3} J_{\mathrm{HH}}=6.9 \mathrm{~Hz}, 2 \mathrm{H}\right), 0.85\left(\mathrm{t},{ }^{3} J_{\mathrm{HH}}=7.1\right.$ $\mathrm{Hz}, 3 \mathrm{H})$. For $(\mathrm{Z})$ isomer, allylic proton appears at $2.46 \mathrm{ppm}(\mathrm{t}$, $\left.{ }^{3} J_{\mathrm{HH}}=7.2 \mathrm{~Hz}, 2 \mathrm{H}\right) .{ }^{13} \mathrm{C} \mathrm{NMR}\left(\mathrm{CDCl}_{3}\right): \delta 159.1,142.8,138.6$, $135.7,128.9,128.3,127.8,126.6\left(\mathrm{t},{ }^{1} J_{\mathrm{CD}}=23.2 \mathrm{~Hz}\right), 126.4$, 113.9, 55.4, 31.2, 30.0, 22.9, 14.0. Anal. Calcd for $\mathrm{C}_{19} \mathrm{H}_{21} \mathrm{DO}$ : C, 85.35; H, 8.67. Found: C, 85.55; H, 8.44.

\section{Procedure for Equation 1}

A solution of $\mathrm{Fe}(\mathrm{acac})_{3}(3.5 \mathrm{mg}, 9.9 \mu \mathrm{mol})$ and $\operatorname{IPr}(15.6 \mathrm{mg}, 40.0 \mu \mathrm{mol})$ in THF (1.0 $\mathrm{mL}$ ) was stirred at room temperature for $5 \mathrm{~min}$. 4-Methoxy-1-phenyl-1-butyne (4, $32.0 \mathrm{mg}, 0.200 \mathrm{mmol}$ ) and 4-methylphenylmagnesium bromide (2n, $0.40 \mathrm{~mL}, 0.40$ mmol; $1.00 \mathrm{M}$ solution in THF) were added, and the mixture was stirred at $60{ }^{\circ} \mathrm{C}$ for 16 h. The reaction was quenched with water $(80 \mu \mathrm{L})$, passed through a pad of silica gel with $\mathrm{Et}_{2} \mathrm{O}$, and concentrated under vacuum. The residue was purified by silica gel preparative TLC with Hexane/ $\mathrm{Et}_{2} \mathrm{O}(95 / 5)$ to give the hydroarylation products (5 and $\mathbf{6}$, $40.8 \mathrm{mg}, 0.161 \mathrm{mmol} ; 81 \%$ yield). 
5. (E)- and (Z)-4-Methoxy-1-2-(4-methylphenyl)-phenyl-1-butene (Colorless oil)

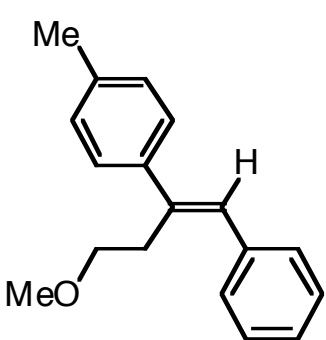

$(E)$

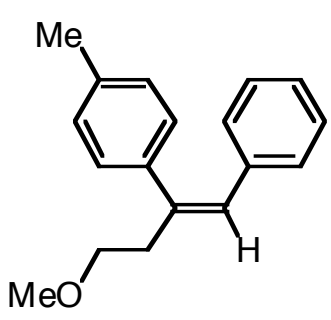

$(Z)$

$(E)-5 ;{ }^{1} \mathrm{H}$ NMR $\left(\mathrm{CDCl}_{3}\right): \delta$ 7.40-7.32 (m, $6 \mathrm{H}), 7.26-7.21(\mathrm{~m}, 1 \mathrm{H}), 7.22\left(\mathrm{~d},{ }^{3} J_{\mathrm{HH}}=7.6\right.$ $\mathrm{Hz}, 2 \mathrm{H}), 6.77(\mathrm{~s}, 1 \mathrm{H}), 3.43\left(\mathrm{t},{ }^{3} J_{\mathrm{HH}}=7.5\right.$ $\mathrm{Hz}, 2 \mathrm{H}), 3.27(\mathrm{~s}, 3 \mathrm{H}), 3.01\left(\mathrm{t},{ }^{3} J_{\mathrm{HH}}=7.5\right.$ $\mathrm{Hz}, \quad 2 \mathrm{H}), \quad 2.37 \quad(\mathrm{~s}, \quad 3 \mathrm{H}) . \quad{ }^{13} \mathrm{C}$ NMR $\left(\mathrm{CDCl}_{3}\right): \delta 139.8,138.1,137.8,137.2$, $129.3,129.2,128.9,128.6,128.4,126.5$, 71.3, 58.7, 30.8, 21.2. (Z)-5; ${ }^{1} \mathrm{H}$ NMR $\left(\mathrm{CDCl}_{3}\right): \delta 7.13-7.02(\mathrm{~m}, 7 \mathrm{H}), 6.94\left(\mathrm{~d},{ }^{3} J_{\mathrm{HH}}\right.$ $=7.9 \mathrm{~Hz}, 2 \mathrm{H}), 6.47(\mathrm{~s}, 1 \mathrm{H}), 3.42\left(\mathrm{t},{ }^{3} J_{\mathrm{HH}}=\right.$ $7.0 \mathrm{~Hz}, 2 \mathrm{H}), 3.30(\mathrm{~s}, 3 \mathrm{H}), 2.75\left(\mathrm{td},{ }^{3} J_{\mathrm{HH}}=7.0 \mathrm{~Hz}\right.$ and $\left.{ }^{4} J_{\mathrm{HH}}=1.2 \mathrm{~Hz}, 2 \mathrm{H}\right), 2.34(\mathrm{~s}, 3 \mathrm{H})$. ${ }^{13} \mathrm{C}$ NMR $\left(\mathrm{CDCl}_{3}\right): \delta 139.8,139.0,137.5,136.8,129.6,129.4,127.9,127.8,126.8$, 126.3, 71.1, 58.7, 40.9, 21.4. Anal. Calcd for $\mathrm{C}_{18} \mathrm{H}_{20} \mathrm{O}: \mathrm{C}, 85.67 ; \mathrm{H}, 7.99$. Found: C, $85.87 ; \mathrm{H}, 7.96$.

\section{Procedures for Equation 2}

To a solution of $\mathrm{Fe}(\mathrm{acac})_{3}(3.5 \mathrm{mg}, 9.9 \mu \mathrm{mol})$ and $\mathrm{IPr}(15.6 \mathrm{mg}, 40.0 \mu \mathrm{mol})$ in THF $(1.0 \mathrm{~mL})$, 1-phenyl-1-hexyne (1a, $31.6 \quad \mathrm{mg}, \quad 0.200 \quad \mathrm{mmol})$ and 4-methoxyphenylmagnesium bromide $(\mathbf{2 m}, 0.38 \mathrm{~mL}, 0.40 \mathrm{mmol} ; 1.06 \mathrm{M}$ solution in THF) were added, and the mixture was stirred at $60{ }^{\circ} \mathrm{C}$ for $16 \mathrm{~h}$. Then allyl bromide (73 $\mathrm{mg}, 0.60 \mathrm{mmol}$ ) was added and the resulting mixture was further stirred at $60{ }^{\circ} \mathrm{C}$ for $2 \mathrm{~h}$. The mixture was passed through a pad of silica gel with $\mathrm{Et}_{2} \mathrm{O}$, and concentrated under vacuum. The residue was purified by silica gel preparative TLC with Hexane $/ \mathrm{Et}_{2} \mathrm{O}(95 / 5)$ to give the product (7, $47.8 \mathrm{mg}, 0.156 \mathrm{mmol} ; 78 \%$ yield).

7. 5-(4-Methoxyphenyl)-4-phenyl-1,4-nonadiene (Pale yellow oil)

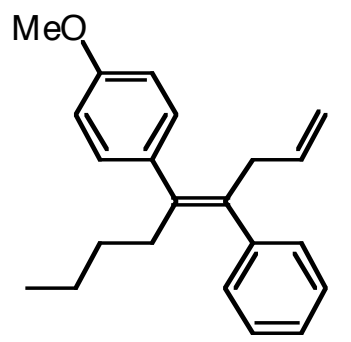

(E)-7; ${ }^{1} \mathrm{H}$ NMR $\left(\mathrm{CDCl}_{3}\right): \delta 7.33\left(\mathrm{t},{ }^{3} J_{\mathrm{HH}}=7.5 \mathrm{~Hz}, 2 \mathrm{H}\right), 7.24(\mathrm{t}$, $\left.{ }^{3} J_{\mathrm{HH}}=7.5 \mathrm{~Hz}, 1 \mathrm{H}\right), 7.19\left(\mathrm{dd},{ }^{3} J_{\mathrm{HH}}=8.2 \mathrm{~Hz}\right.$ and $\left.{ }^{4} J_{\mathrm{HH}}=1.2 \mathrm{~Hz}, 2 \mathrm{H}\right)$, $7.15\left(\mathrm{~d},{ }^{3} J_{\mathrm{HH}}=8.8 \mathrm{~Hz}, 2 \mathrm{H}\right), 6.90\left(\mathrm{~d},{ }^{3} J_{\mathrm{HH}}=8.5 \mathrm{~Hz}, 2 \mathrm{H}\right), 5.62(\mathrm{ddt}$, ${ }^{3} J_{\mathrm{HH}}=17.0 \mathrm{~Hz},{ }^{3} J_{\mathrm{HH}}=10.0 \mathrm{~Hz}$ and $\left.{ }^{3} J_{\mathrm{HH}}=6.7 \mathrm{~Hz}, 1 \mathrm{H}\right), 4.83(\mathrm{dd}$, ${ }^{3} J_{\mathrm{HH}}=10.0 \mathrm{~Hz}$ and $\left.{ }^{2} J_{\mathrm{HH}}=2.1 \mathrm{~Hz}, 1 \mathrm{H}\right), 4.80\left(\mathrm{dd},{ }^{3} J_{\mathrm{HH}}=17.0 \mathrm{~Hz}\right.$ and $\left.{ }^{2} J_{\mathrm{HH}_{3}}=2.1 \mathrm{~Hz}, 1 \mathrm{H}\right), 3.83(\mathrm{~s}, 3 \mathrm{H}), 2.93\left(\mathrm{~d},{ }^{3} \mathrm{~J}_{\mathrm{HH}}=6.4 \mathrm{~Hz}, 2 \mathrm{H}\right)$, $2.14\left(\mathrm{t},{ }^{3} J_{\mathrm{HH}}=7.6 \mathrm{~Hz}, 2 \mathrm{H}\right), 1.20-1.06(\mathrm{~m}, 4 \mathrm{H}), 0.70\left(\mathrm{t},{ }^{3} J_{\mathrm{HH}}=7.2\right.$ $\mathrm{Hz}, 3 \mathrm{H})$. For $(Z)$ isomer, allylic proton appears at $3.30 \mathrm{ppm}(\mathrm{d}$, $\left.{ }^{3} J_{\mathrm{HH}}=6.1 \mathrm{~Hz}, 2 \mathrm{H}\right)$ and $2.52 \mathrm{ppm}\left(\mathrm{t},{ }^{3} J_{\mathrm{HH}}=7.5 \mathrm{~Hz}, 2 \mathrm{H}\right) .{ }^{13} \mathrm{C}$ $\operatorname{NMR}\left(\mathrm{CDCl}_{3}\right): \delta 158.3,142.9,139.4,136.8,135.7,134.9,129.8,129.0,128.0,126.4$, 115.3, 113.6, 55.3, 40.5, 35.4, 30.9, 22.6, 14.0. Anal. Calcd for $\mathrm{C}_{22} \mathrm{H}_{26} \mathrm{O}: \mathrm{C}, 86.23 ; \mathrm{H}$, 8.55. Found: C, 85.97; H, 8.41.

1-Phenyl-1-hexyne (1a, 31.6 $\mathrm{mg}, 0.200 \mathrm{mmol}$ ) and 4-methoxyphenylmagnesium bromide $(2 \mathrm{~m}, 0.38 \mathrm{~mL}, 0.40 \mathrm{mmol} ; 1.06 \mathrm{M}$ solution in THF) were added to a solution of $\mathrm{Fe}(\mathrm{acac})_{3}(3.5 \mathrm{mg}, 9.9 \mu \mathrm{mol})$ and $\mathrm{IPr}(15.6 \mathrm{mg}, 40.0 \mu \mathrm{mol})$ in THF $(1.0 \mathrm{~mL})$. After stirring at $60{ }^{\circ} \mathrm{C}$ for $16 \mathrm{~h}, \mathrm{ZnCl}_{2}(73 \mathrm{mg}, 0.60 \mathrm{mmol})$ was added and the mixture was stirred at room temperature for $5 \mathrm{~min}$, and then $\mathrm{I}_{2}(152 \mathrm{mg}, 0.599 \mathrm{mmol})$ was added. The resulting mixture was further stirred at room temperature for $2 \mathrm{~h}$, passed through a pad of silica gel with $\mathrm{Et}_{2} \mathrm{O}$, and concentrated under vacuum. The residue was purified by silica gel preparative TLC with Hexane/ $\mathrm{Et}_{2} \mathrm{O}(95 / 5)$ to give the alkenyl iodide $(8$, $71.4 \mathrm{mg}, 0.182 \mathrm{mmol}$; $91 \%$ yield). 
8. 1-Iodo-2-(4-methoxyphenyl)-1-phenyl-1-hexene (Pale yellow oil)

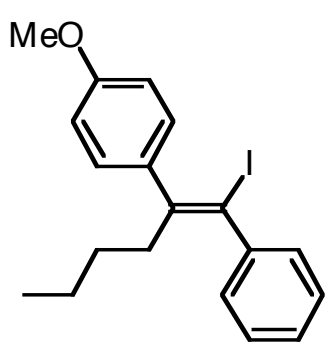

(E)-8; ${ }^{1} \mathrm{H}$ NMR $\left(\mathrm{CDCl}_{3}\right): \delta$ 7.36-7.32 (m, 4H), 7.27-7.23 (m, $\left.1 \mathrm{H}\right)$, $7.17\left(\mathrm{~d},{ }^{3} J_{\mathrm{HH}}=7.9 \mathrm{~Hz}, 2 \mathrm{H}\right), 6.93\left(\mathrm{~d},{ }^{3} J_{\mathrm{HH}}=8.5 \mathrm{~Hz}, 2 \mathrm{H}\right), 3.84(\mathrm{~s}$, $3 \mathrm{H}), 2.30\left(\mathrm{t},{ }^{3} J_{\mathrm{HH}}=7.4 \mathrm{~Hz}, 2 \mathrm{H}\right), 1.20$ (quint, $\left.{ }^{3} J_{\mathrm{HH}}=7.5 \mathrm{~Hz}, 2 \mathrm{H}\right)$, 1.11 (sextet, $\left.{ }^{3} J_{\mathrm{HH}}=7.5 \mathrm{~Hz}, 2 \mathrm{H}\right), 0.70\left(\mathrm{t},{ }^{3} J_{\mathrm{HH}}=7.3 \mathrm{~Hz}, 3 \mathrm{H}\right)$. For $(Z)$ isomer, allylic proton appears at $2.79 \mathrm{ppm}\left(\mathrm{t},{ }^{3} J_{\mathrm{HH}}=7.3 \mathrm{~Hz}\right.$, $2 \mathrm{H}) .{ }^{13} \mathrm{C} \mathrm{NMR}\left(\mathrm{CDCl}_{3}\right): \delta 158.9,150.0,144.6,138.5,129.5$, 128.7, 128.4, 127.8, 113.7, 97.7, 55.3, 35.4, 30.7, 22.3, 13.8. Anal. Calcd for $\mathrm{C}_{19} \mathrm{H}_{21} \mathrm{IO}: \mathrm{C}, 58.17 ; \mathrm{H}, 5.40$. Found: C, 58.40; H, 5.54 .

\section{Procedure for Equation 3}

A solution of $\mathrm{Fe}(\mathrm{acac})_{3}(3.5 \mathrm{mg}, 9.9 \mu \mathrm{mol})$ and $\operatorname{IPr}(15.6 \mathrm{mg}, 40.0 \mu \mathrm{mol})$ in THF $(1.0$ $\mathrm{mL})$ was stirred at room temperature for $5 \mathrm{~min}$, and 4-methoxy-1-phenyl-1-butyne (4, $32.0 \mathrm{mg}, 0.200 \mathrm{mmol}$ ) and 4-methylphenylmagnesium bromide (2n, $0.40 \mathrm{~mL}, 0.40$ mmol; $1.00 \mathrm{M}$ solution in THF) were added. The mixture was stirred at $60{ }^{\circ} \mathrm{C}$ for $16 \mathrm{~h}$ before a suspension of $\mathrm{NiCl}_{2}\left(\mathrm{PPh}_{3}\right)_{2}(6.5 \mathrm{mg}, 9.9 \mu \mathrm{mol})$ and iodobenzene $(122 \mathrm{mg}$, $0.598 \mathrm{mmol})$ in THF $(0.5 \mathrm{~mL})$ was added. The mixture was further stirred at $60{ }^{\circ} \mathrm{C}$ for $16 \mathrm{~h}$, and it was passed through a pad of silica gel with $\mathrm{Et}_{2} \mathrm{O}$, and concentrated under vacuum. The residue was purified by silica gel preparative TLC with $\mathrm{Hexane} / \mathrm{Et}_{2} \mathrm{O}$ $(95 / 5)$ to give the product $(\mathbf{9}, 47.0 \mathrm{mg}, 0.143 \mathrm{mmol} ; 72 \%$ yield $)$.

9. 4-Methoxy-2-(4-methylphenyl)-1,1-diphenyl-1-butene (White solid)

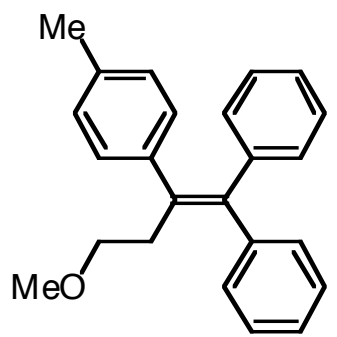

${ }^{1} \mathrm{H} \mathrm{NMR}\left(\mathrm{CDCl}_{3}\right): \delta 7.33\left(\mathrm{t},{ }^{3} J_{\mathrm{HH}}=7.3 \mathrm{~Hz}, 2 \mathrm{H}\right), 7.28\left(\mathrm{~d},{ }^{3} J_{\mathrm{HH}}=7.0\right.$ $\mathrm{Hz}, 2 \mathrm{H}), 7.24\left(\mathrm{t},{ }^{3} \mathrm{~J}_{\mathrm{HH}}=7.3 \mathrm{~Hz}, 1 \mathrm{H}\right), 7.04-6.93(\mathrm{~m}, 7 \mathrm{H}), 6.89(\mathrm{~d}$, $\left.{ }^{3} J_{\mathrm{HH}}=7.0 \mathrm{~Hz}, 2 \mathrm{H}\right), 3.31\left(\mathrm{t},{ }^{3} J_{\mathrm{HH}}=7.3 \mathrm{~Hz}, 2 \mathrm{H}\right), 3.20(\mathrm{~s}, 3 \mathrm{H}), 2.75$ $\left(\mathrm{t},{ }^{3} J_{\mathrm{HH}}=7.3 \mathrm{~Hz}, 2 \mathrm{H}\right), 2.26(\mathrm{~s}, 3 \mathrm{H}) .{ }^{13} \mathrm{C} \mathrm{NMR}\left(\mathrm{CDCl}_{3}\right): \delta 143.3$, $143.2,141.0,139.0,136.7,136.0,130.8,129.7,129.5,128.8$, 128.3, 127.5, 126.8, 125.9, 71.4, 58.6, 36.1, 21.3. Anal. Calcd for $\mathrm{C}_{24} \mathrm{H}_{24} \mathrm{O}$ : C, 87.76; H, 7.37. Found: C, 87.46; H, 7.37. 